\title{
A Post-Newtonian Treatment of Relativistic Compact Object Binaries in Star Clusters
}

\author{
J. M. B. Downing and R. Spurzem \\ Astronomisches Rechen-Institut, Zentrum für Astronomie, Universität Heidelberg \\ email: downin@ari.uni-heidelberg.de
}

\begin{abstract}
Stellar mass compact object binaries are promising sources of gravitational radiation for the current generation of ground-based detectors, VIRGO and LIGO. Accurate templates for gravitational waveforms are needed in order to extract an event from the VIRGO/LIGO data stream. In the case of relativistic, compact object binaries accurate orbital parameters are necessary in order to produce such templates. Binary systems are affected by their stellar environment and thus the parameters of the binary population of a dense star cluster will be different from those of the field population. We propose to investigate the parameters of relativistic binary populations in dense star clusters using direct N-body simulations with a Post-Newtonian treatment of general relativity for the close binaries.
\end{abstract}

Keywords. stellar dynamics, relativity, gravitational waves, methods: n-body simulations, binaries: close

\section{Introduction}

Compact object binaries in the relativistic regime are usually assumed to be circularised by the emission of gravitational radiation and this assumption goes into the production of gravitational wave templates and estimates of relativistic merger event rates. It is known, however, that orbital parameters of compact object binaries can have significant effects on the gravitational radiation. In particular an eccentricity of $0.8-0.9$ can produce an enhancement of $10^{2}-10^{3}$ over a circular orbit with the same energy (Peters \& Mathews 1963). Since this effect occurs primarily at periastron it is possible that eccentric binaries are capable of emitting significant gravitational radiation at larger separations than circularised binaries. Hard binaries with high eccentricities exist in dense star clusters and we propose to examine the relativistic segment of this population.

\section{The NBODY6++ Code and the PN Formalism}

To perform our direct N-body integration we use the code NBODY6++. This code is part of the series of NBODY codes (Aarseth 1999) modified for operation on parallel machines (Spurzem 1999). The code features a fourth-order Hermite predictor-corrector scheme, parallel operation, individual and block time stepping, an Ahmad-Cohen neighbour scheme (Ahmad \& Cohen 1973), and Kustaanheimo and Stiefel (KS) regularisation for binaries (Kustaanheimo \& Stiefel 1965). The KS regularisation rotates the Kepler problem through quaternion space into a simple harmonic oscillator. Other forces, such as Post-Newtonian corrections for relativistic motion, can then be applied as perturbations to the harmonic oscillator. 


\section{The Post-Newtonian Formalism}

The Post-Newtonian formalism corrects the classical Newtonian acceleration with a series expansion in orders of $v / c$ (Blanchet 2006):

$G^{\alpha \beta}\left[g, \partial g, \partial^{2} g\right]=\frac{8 \pi G}{c^{4}} T^{\alpha \beta}[g] \quad \Longrightarrow \quad \vec{a}=\overrightarrow{a_{N}}+\frac{v}{c} \overrightarrow{a_{1}}+\frac{v^{2}}{c^{2}} \overrightarrow{a_{2}}+\frac{v^{3}}{c^{3}} \overrightarrow{a_{3}}+\frac{v^{4}}{c^{4}} \overrightarrow{a_{4}}+\frac{v^{5}}{c^{5}} \overrightarrow{a_{5}}+\ldots$

The first- and third-order accelerations vanish and the remaining terms are referred to by their relative order. The effect of the Post-Newtonian terms can be enhanced in a controlled manner by decreasing the velocity of light in the expansion with respect to the characteristic velocity in the N-body system. This allows us to produce more events per unit time for better statistics.

\section{Current Work and Outlook}

The Post-Newtonian expansion up to PN2.5 was previously incorporated into NBODY6 (single processor) by Kupi, Amaro-Seoane \& Spurzem (2006). We have now incorporated the same expansion into NBODY6++ (multiple processor version) and are currently testing the code. We have some initial physical results such as number of mergers as a function of time (Fig. 1) but more work is needed before these can be meaningfully interpreted. One challenge is that there are no terms for the Post-Newtonian energies that are

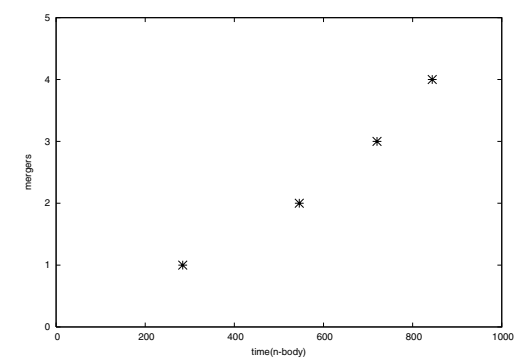

Figure 1. Number of relativistic merges per unit time for an $8 \mathrm{k}$ particle simulation with no primordial binaries. $c$ is chosen so the the relativistic terms are enhanced by a factor of $\sim 300$.

conserved at each order, causing difficulty in monitoring the overall energy conservation of the code. Once this problem is solved we intend to start simulations with realistic numbers of primordial binaries using the Kyoto II initial conditions in order to produce data that can be used for the production of gravitational wave templates.

\section{Acknowledgements}

J.M.B. Downing would like to acknowledge the IMPRS-Astronomy, Heidelberg for funding his Ph.D. Thesis.

\section{References}

Aarseth, S. J. 1999, PASP 111, 1333

Ahmad, A. \& Cohen, L. 1973, J. Comput. Phys. 12, 389

Blanchet, L. 2006, Living Rev. Relativity 9, 4,

Kupi, G., Amaro-Seoane, P., \& Spurzem, R. 2006, MNRAS 371, L45

Kustaanheimo, P. \& Stiefel, E. 1965, J. Reine Angew. Math. 218, 204

Peters, P. C. \& Mathews, J. 1977, Phys. Rev. 131 no. 1, 435

Spurzem, F. 1999, J. Comp. Appl. Maths. 109, 407 